\title{
Cobalt and Nitrogen Co-Doped Nano-Porous Carbon: Synthesis and Application for Lithium-Sulfur Battery
}

\author{
Shiqiang Luo, Chunman Zheng, Yujie Li, Shuangke Liu \\ College of Aerospace Science and Engineering, National University of Defense Technology, Changsha, China \\ Email: zhengchunman@nudt.edu.cn
}

How to cite this paper: Luo, S.Q., Zheng, C.M., Li, Y.J. and Liu, S.K. (2017) Cobalt and Nitrogen Co-Doped Nano-Porous Carbon: Synthesis and Application for Lithium-Sulfur Battery. Journal of Power and Energy Engineering, 5, 16-20.

https://doi.org/10.4236/jpee.2017.512003

Received: November 1, 2017

Accepted: November 14, 2017

Published: November 21, 2017

\begin{abstract}
S@C-Co-N nanoporous carbon co-doped with cobalt and nitrogen as the cathode of lithium-sulfur battery are prepared. The synthetic route is carried out via the carbonization of metal organic frameworks polyhedron ZIF-67, followed by the heat treatment with sulfur. The SEM images suggest that C-Co-N composite maintains almost the same size and polyhedron shape of ZIF-67. The XRD pattern confirms the existence of cobalt element. As cathode for lithium-sulfur battery, the S@C-Co-N composite delivers a reversible capacity of $916.6 \mathrm{mAh} \cdot \mathrm{g}^{-1}$ at the initial cycle and $460.5 \mathrm{mAh} \cdot \mathrm{g}^{-1}$ after 500 cycles at $0.5 \mathrm{C}$, with a capacity fading of $0.09 \%$ per cycle.
\end{abstract}

\section{Keywords}

Metal-Organic Frameworks, Lithium-Sulfur Battery, Cathode

\section{Introduction}

Metal-organic frameworks (MOFs) are a class of porous materials [1] that have attracted enormous attention during the past two decades due to their high surface areas, controllable structures and tunable pore sizes [2] [3]. Benefiting from the advantageous features of MOFs, numerous MOF-derived materials with various porous structures and compositions, such as spherical/non-spherical hollow materials, and hierarchical carbon structures, have been successfully prepared in recent years through various synthetic approaches. Recently, there has been a rapidly growing interest in developing MOF-based materials [4] for electrochemical energy storage [5]. Lithium-sulfur batteries have a high energy density $(2600 \mathrm{Wh} / \mathrm{kg})[6]$ and theoretical specific capacity $\left(1675 \mathrm{mAh} \cdot \mathrm{g}^{-1}\right)$ and 
rely on cheap and environmentally friendly sulfur cathode materials. However, a volume change as high as $80 \%$ is accompanied with the reaction [7]. Moreover, both the sulfur itself and $\mathrm{Li}_{2} \mathrm{~S}$ are insulators, [8] which require the incorporation of conductive additives and thus lead to the poor utilization of active material. Additionally, polysulfides shuttle effect [9] [10] remains a significant challenges to practical applications of the Lithium-sulfur batter. Therefore, storage and immobilization of dissolved polysulfides is of great importance to avoid low coulombic efficiency and self-discharge behavior in designing a Li-S battery with long cycle life. MOF-derived carbon materials have shown high surface area, hierarchically porous structures and good electrical conductivity, making them attractive candidates for Li-S batteries. In this work, a well-designed 3D porous carbon structure co-doped with cobalt and nitrogen (C-Co-N) derived from the direct carbonization of metal organic frameworks polyhedron ZIF-67 was prepared [11] to host sulfur. When evaluated as the cathode of lithium-sulfur battery, it shows enhanced cycle stability.

\section{Experimental Section}

\subsection{Synthesis of Materials}

Synthesis of ZIF-67nanocrystals: 2-methylimidazole were purchased from Aladdin, $\mathrm{Co}\left(\mathrm{NO}_{3}\right)_{2} \cdot 6 \mathrm{H}_{2} \mathrm{O}$ and methanol were purchased from Sinopharm and used without further purification. In a typical synthesis, $2.328 \mathrm{~g}$ of $\mathrm{Co}\left(\mathrm{NO}_{3}\right)_{2} \cdot 6 \mathrm{H}_{2} \mathrm{O}$ and $2.627 \mathrm{~g}$ of 2-methylimidazole were dissolved in the $100 \mathrm{~mL}$ of methanol solution. Then 2-methylimidazole solution was slowly added into $\mathrm{Co}\left(\mathrm{NO}_{3}\right)_{2} \cdot 6 \mathrm{H}_{2} \mathrm{O}$ solution under string. Then the mixed solution was kept at room temperature for $24 \mathrm{~h}$. The obtained precipitates were collected by centrifugation, washed with ethanol at least three times, and finally dried under vacuum at $80^{\circ} \mathrm{C}$ for $12 \mathrm{~h}$.

Synthesis of C-Co-N nanoporous carbon: The as-prepared ZIF-67 polyhedrons were annealed in a tube furnace under an $\mathrm{Ar}$ flow at $700^{\circ} \mathrm{C}$ for $3 \mathrm{~h}$, with a heating rate of $5^{\circ} \mathrm{C} / \mathrm{min}$, and then naturally cooled down to room temperature, the $\mathrm{C}-\mathrm{Co}-\mathrm{N}$ composites were obtained.

Synthesis of S@C-Co-N composites: The prepared C-Co-N composites is mixed with sulfur with a mass ratio of $\mathrm{Ms}$ : $\mathrm{Mc}=7: 3$, milling for $30 \mathrm{~min}$ and sealed in the tube furnace heated at $155^{\circ} \mathrm{C}$ for $10 \mathrm{~h}$, and then further treated at $210^{\circ} \mathrm{C}$ for $10 \mathrm{~min}$. The S@C-Co-N cathode materials were obtained.

\subsection{Electrochemical Measurements}

The electrochemical performances were measured using coin-type 2016 cells, which are assembled in the argon-filled glove box $\left(\mathrm{H}_{2} \mathrm{O}, \mathrm{O}_{2}<1 \mathrm{ppm}\right)$. The working electrode is prepared by mixing the S@C-Co-N composites, super $\mathrm{p}$ carbon and water-soluble polymer-lauryl acrylate (LA133) binder in a weight ratio of 80:10:10 in water solvent. Galvanostatic charge-discharge cycles were tested with a LANDCT2001A instrument at various C-rates between 1.7 and 2.8 $\mathrm{V}\left(\mathrm{vs} \mathrm{Li}^{+} / \mathrm{Li}\right)$ at constant temperature of $25^{\circ} \mathrm{C}$. 


\section{Result and Discussion}

SEM image shows that ZIF-67 polyhedrons with the uniform size of about 500 $\mathrm{nm}$. After carbonization process, the ZIF-67 precursor transformed to the C-Co-N. Figure 1 suggests that $\mathrm{C}-\mathrm{Co}-\mathrm{N}$ composites maintain almost the same size and polyhedron shape of ZIF-67.

The crystal structures of the as-prepared ZIF-67 and C-Co-N composites were confirmed by X-ray diffraction (XRD). After carbonization process in argon atmosphere, the 2-methylimidazole ligands transform to the $\mathrm{N}$-doped porous carbon polyhedrons, meanwhile, the $\mathrm{Co}^{2+}$ is reduced to Co nanoparticles, the XRD patterns (Figure 2) show the existence of the Co nanoparticles, the characteristic peaks of C-Co-N composite perfectly corresponding to the JCPDS card of Co crystal (PDF\#15-0808). In addition, the homogeneous distribution of cobalt nanoparticles and the nitrogen group in the $\mathrm{C}-\mathrm{Co}-\mathrm{N}$ composite not only significantly protect soluble polysulfide from migrating out of the framework through strong chemical interactions but also facilitate the catalytic effect in the charge and discharge of Li-S batteries.

As shown in Figure 3, the S@C-Co-N cathode delivers an initial specific capacity of $916.6 \mathrm{mAh} \cdot \mathrm{g}^{-1}$ at $0.5 \mathrm{C}$, which is stabilized after the 30 th cycle. After 500 cycles, the capacity remains at $460.5 \mathrm{mAh} \cdot \mathrm{g}^{-1}$, corresponding to as low fading rate of $0.09 \%$ percycle. $\mathrm{C}-\mathrm{Co}-\mathrm{N}$ carbon structure displays a good long cycle stability. The rate capability of the S@C-Co-N cathode was conducted at $0.1-2$ C-rates. The S@C-Co-N cathode exhibits a better rate capability (Figure 4) with high reversible specific capacities of $1030,847,728$ and $372.7 \mathrm{mAh} \cdot \mathrm{g}^{-1}$ at $0.1 \mathrm{C}$, $0.2 \mathrm{C}, 0.5 \mathrm{C}, 1 \mathrm{C}$ and $2 \mathrm{C}$. Importantly, the capacity recovers to $870.2 \mathrm{mAh} \cdot \mathrm{g}^{-1}$ as the $\mathrm{C}$-rate is reduced from 2 to $0.1 \mathrm{C}$, indicating the stable structure of the
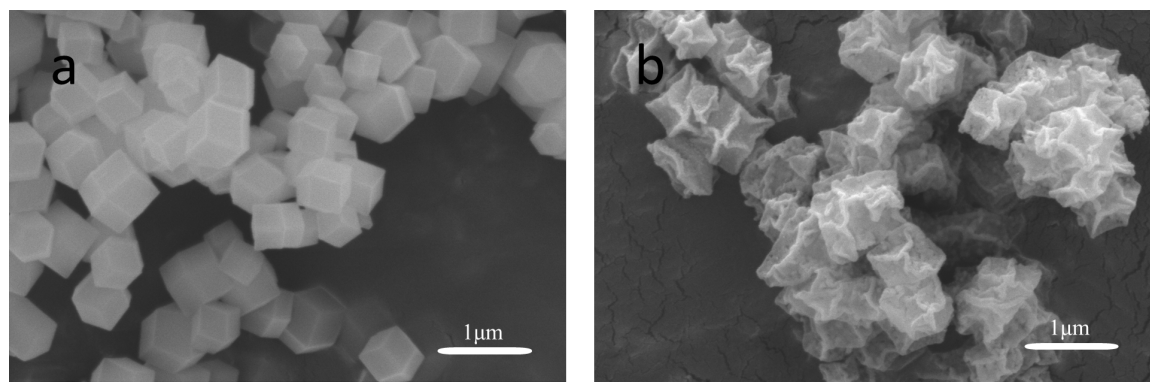

Figure 1. SEM image of ZIF-67 (a) and C-Co-N nanoporous carbon (b).

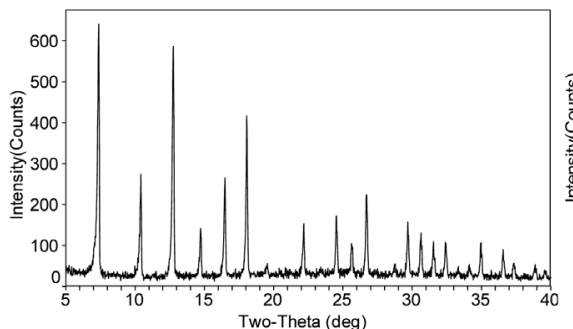

(a)

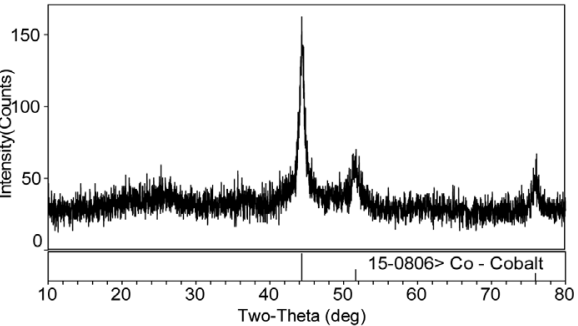

(b)

Figure 2. XRD patterns of ZIF-67 (a) and C-Co-N nanoparticles (b). 


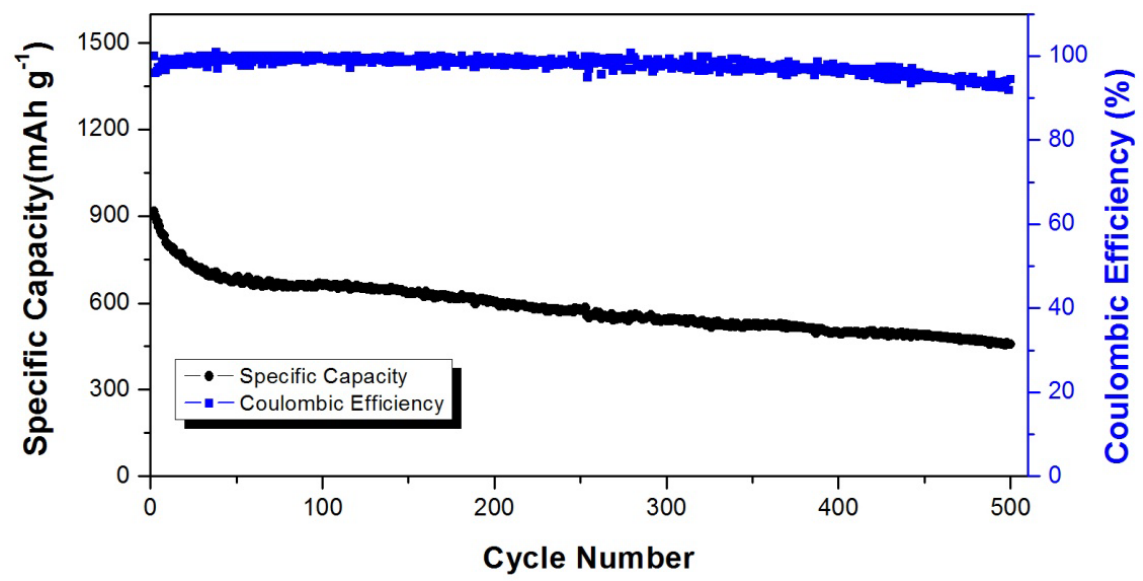

Figure 3. Cyclic performance of the S@C-Co-N cathode at $0.5 \mathrm{C}$ for 500 cycles.

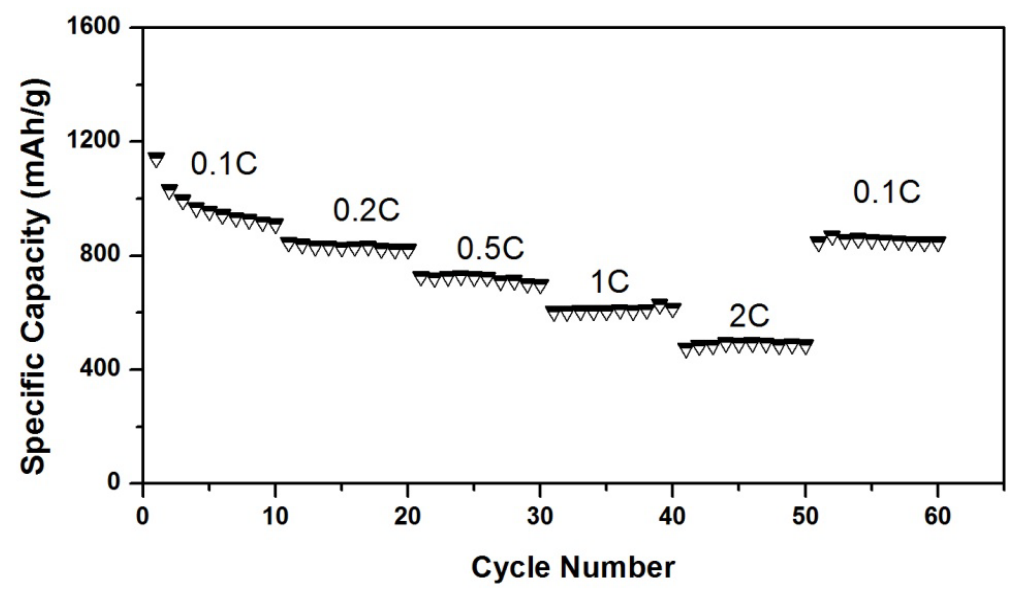

Figure 4. Rate performances at 0.1 - 2 C-rates of the S@C-Co-N cathode.

S@C-Co-N electrode in a high rate cycling. Such cycling stability and capacity reversibility of S@C-Co-N are mainly attributed to the doped nitrogen group in the carbon matrix, which can confine polysulfide species by the strong interaction [12] of $\mathrm{Li}$ in $\mathrm{Li}_{2} \mathrm{~S}_{\mathrm{n}}$ with $\mathrm{N}$ atoms. The doped $\mathrm{N}$ can facilitate the oxidation of $\mathrm{Li}_{2} \mathrm{~S}_{6} \rightarrow \mathrm{Li}_{2} \mathrm{~S}_{8} \rightarrow \mathrm{S}_{8}$, leading to the improved cycle performance [13].

\section{Conclusion}

In summary, a well-designed 3D porous carbon matrix (C-Co-N) derived from an MOF polyhedron (ZIF-67) was prepared to host sulfur. Due to the crystalline configuration and abundant micro-porous, the C-Co-N architecture not only ensures sufficient space to host sulfur nanoparticles but also efficiently prevents polysulfides from dissolution by physical interaction. Furthermore, the existence of Co nanoparticles and doped nitrogen in the S@C-Co-N composite effectively confines the soluble polysulfides by chemical interaction, and the catalytic effect resulting from the Co nanoparticles' distribution also plays an important role during the cycling process. At all, the use of MOF-derived materials in electrode materials maybe a good research point. 


\section{References}

[1] Furukawa, H., Cordova, K.E., O’Keeffe, M. and Yaghi, O.M. (2013) The Chemistry and Application of Metal-Organic Frameworks. Science, 341, 974-988. https://doi.org/10.1126/science.1230444

[2] Eddaoudi, M., Kim, J., Rosi, N., Vodak, D., Wachter, J., O’Keefe, M. and Yaghi, O.M. (2002) Systematic Design of Pore Size and Functionality in Isoreticular MOFs and Their Application in Methane Storage. Science, 295, 469-472. https://doi.org/10.1126/science.1067208

[3] Wang, Z. and Cohen, S.M. (2007) Postsynthetic Covalent Modification of a Neutral Metal-Organic Framework. Journal of the American Chemical Society, 129, 12368-12369. https://doi.org/10.1021/ja074366o

[4] Combarieu, G.D., Hamelet, S., Millange, F., Morcrette, M., Tarascon J.M., Ferey, G. and Walton, R.I. (2009) In Situ Fe XAFS of Reversible Lithium Insertion in a Flexible Metal Organic Framework Material. Electrochemistry Communications, 11, 1881-1884. https://doi.org/10.1016/j.elecom.2009.08.008

[5] Xia, W., Mahmood, A., Zou, R.Q. and Xu, Q. (2015) Metal-Organic Frameworks and Their Derived Nanostructures for Electrochemical Energy Storage and Conversion. Energy \& Environmental Science, 8, 1837-1866. https://doi.org/10.1039/C5EE00762C

[6] Ji, L.W., Rao, M.M., Zheng, H.M., Zhang, L., Li, Y.C., Duan, W.H., Guo, J.H., Cairns, E.J. and Zhang, Y.G. (2011) Graphene Oxide as a Sulfur Immobilizer in High Performance Lithium/Sulfur Cells. Journal of the American Chemical Society, 133, 18522. https://doi.org/10.1021/ja206955k

[7] Zhang, K., Xu, Y., Lu, Y., Zhu, Y., Qian, Y., Wang, D., Zhou, J., Lin, N. and Qian, Y.A. (2016) A Graphene Oxide-Wrapped Bipyramidal Sulfur@Polyaniline Core-Shell Structure as a Cathode for Li-S Batteries with Enhanced Electrochemical Performance. Journal of Materials Chemistry A, 4, 6404-6410. https://doi.org/10.1039/C6TA01118G

[8] Manthiram, A., Fu, Y.Z., Chung, S.H., Zu, C.X. and Su, Y.S. (2014) Rechargeable Lithium-Sulfur Batteries. Chemical Reviews, 114, 11751. https://doi.org/10.1021/cr500062v

[9] Ji X.L., Lee, K.T. and Nazar, L.F. (2009) Nanocrystalline Intermetallics on Mesoporous Carbon for Direct Formic Acid Fuel Cell Anodes. Nature Materials, 8, 500. https://doi.org/10.1038/nchem.553

[10] Talapaneni, S.N., Hwang, T.H., Je, S.H., Buyukcakir, O. and Choi, J.W. (2016) Elemental-Sulfur-Mediated Facile Synthesis of a Covalent Triazine Framework for High-Performance Lithium-Sulfur Batteries. Angewandte Chemie International Edition, 55, 3106. https://doi.org/10.1002/anie.201511553

[11] Zhou, J., Lin, N. and Cai, W.L. (2016) Synthesis of S/CoS ${ }_{2}$ Nanoparticles-Embedded N-doped Carbon Polyhedrons from Polyhedrons ZIF-67 and Their Properties in Lithium-Sulfur Batteries. Electrochimica Acta, 218, 243-251. https://doi.org/10.1016/j.electacta.2016.09.130

[12] He, J.R., Chen, Y.F., Lv, W.Q. and Wen, K.C. (2016) From Metal-Organic Framework to $\mathrm{Li}_{2} \mathrm{~S} @ \mathrm{C}-\mathrm{Co}-\mathrm{N}$ Nanoporous Architecture: A High-Capacity Cathode for Lithium-Sulfur Batteries. ACS Nano, 10, 1098-10987.

https://doi.org/10.1021/acsnano.6b05696

[13] Chen, J., Yuan, R., Feng, J., Zhang, Q., Huang, J., Fu, G., Zheng, M., Ren, B. and Dong, Q. (2015) Conductive Lewis Base Matrix to Recover the Missing Link of $\mathrm{Li}_{2} \mathrm{~S}_{8}$ during the Sulfur Redox Cycle in Li-S Battery. Chemistry of Materials, 27, 2048-2055. https://doi.org/10.1021/cm5044667 